\title{
Identifying Behavioral Phenotypes and Heterogeneity in Heart Valve Surface Endothelium
}

\author{
Alicia A. Blancas Liezl R. Balaoing Francisca M. Acosta K. Jane Grande-Allen \\ Department of Bioengineering, Rice University, Houston, Tex., USA
}

\section{Key Words}

Endothelium · Aortic valve · Lymphatic endothelial cells .

Angiogenesis

\begin{abstract}
Heart valvular endothelial cells (VECs) are distinct from vascular endothelial cells (ECs), but have an uncertain context within the spectrum of known endothelial phenotypes, including lymphatic ECs (LECs). Profiling the phenotypes of the heart valve surface VECs would facilitate identification of a proper seeding population for tissue-engineered valves, as well as elucidate mechanisms of valvular disease. Porcine VECs and porcine aortic ECs (AECs) were isolated from pig hearts and characterized to assess known EC and LEC markers. A transwell migration assay determined their propensity to migrate toward vascular endothelial growth factor, an angiogenic stimulus, over $24 \mathrm{~h}$. Compared to AECs, Flt-1 was expressed on almost double the percentage of VECs, measured as 74 versus $38 \%$. The expression of angiogenic EC markers CXCR4 and DLL4 was $>90 \%$ on AECs, whereas VECs showed only $35 \%$ CXCR4+ and 47\% DLL4+. AECs demonstrated greater migration ( $71.5 \pm 11.0$ cells per image field) than the VECs with $30.0 \pm 15.3$ cells per image field $(p=$ 0.032). In total, $30 \%$ of VECs were positive for LYVE1+/Prox 1+, while these markers were absent in AECs. In conclusion, the population of cells on the surface of heart valves is hetero-
\end{abstract}

geneous, consisting largely of nonangiogenic VECs and a subset of LECs. Previous studies have indicated the presence of LECs within the interior of the valves; however, this is the first study to demonstrate their presence on the surface. Identification of this unique endothelial mixture is a step forward in the development of engineered valve replacements as a uniform EC seeding population may not be the best option to maximize transplant success.

(c) 2016 S. Karger AG, Basel

\section{Introduction}

Valvular endothelial cells (VECs) have been identified to have distinct differences in biomechanical responses and gene expression in comparison to endothelial cells

\begin{tabular}{ll}
\hline Abbreviations used in this paper \\
\hline AECs & aortic endothelial cells \\
BSA & bovine serum albumin \\
ECs & endothelial cells \\
EPCs & endothelial progenitor cells \\
LECs & lymphatic endothelial cells \\
PBS & phosphate-buffered saline \\
VECs & valvular endothelial cells \\
VEGF & vascular endothelial growth factor \\
\hline
\end{tabular}

\section{KARGER}

E-Mail karger@karger.com www.karger.com/cto
(C) 2016 S. Karger AG, Basel

$1422-6405 / 16 / 2014-0268 \$ 39.50 / 0$
K. Jane Grande-Allen, $\mathrm{PhD}$

Department of Bioengineering, Rice University 6100 Main Street, MS 142

Houston, TX 77005 (USA)

E-Mail grande@ rice.edu 
(ECs) found in the vascular system. Among the earliest observations that distinguish the biomechanical responses of VECs from that of vascular ECs was their perpendicular alignment to the direction of flow, contrary to the parallel cell alignment characteristic of vascular ECs [Deck, 1986; Butcher et al., 2004]. While both cell types express similar antioxidant and anti-inflammatory genes, VECs were found to demonstrate a lower expression of inflammatory-related genes than vascular ECs [Butcher et al., 2004, 2006]. Additionally, VECs expressed more genes related to cartilage formation, whereas vascular ECs had a greater expression of genes involved in bone formation [Butcher et al., 2006]. One of the key differences between cartilage and bony tissues is their vascularization; cartilage tissues are primarily avascular, whereas bone tissues are highly vascularized and in fact require angiogenesis for bone formation. These comparative results suggest that VECs perform a protective role against calcification [Wiltz et al., 2013; Puperi et al., 2015], even within their dynamic shear stress environment, and that they may not readily express genes for angiogenesis.

Furthermore, even though confluent VEC cultures can demonstrate the cobblestone appearance typical of EC monolayers, VECs appear to be much more sensitive to the presence of various proteins than would be observed in other EC cultures. For example, chemokines transforming growth factor- $\beta$ and tissue necrosis factor- $\alpha$ have both been shown to induce VECs to express a mesenchymal phenotype (elongated instead of cobblestone), to express $\alpha$-smooth muscle actin, an interstitial marker, and to reduce VEC expression of CD31 [Paranya et al., 2001; Paruchuri et al., 2006; Mahler et al., 2013; Farrar and Butcher, 2014]. Therefore, this particular process results in the VECs taking on traits characteristic of valve interstitial cells, which are located in the interior of the valve. This complex biochemical process has been studied in detail, with reports describing some heterogeneity in VEC transdifferentiation responses to various chemokines [Paranya et al., 2001; Paruchuri et al., 2006; ElHamamsy et al., 2010; Farrar and Butcher, 2014]. In addition, previous gene analysis comparisons of VECs isolated from the outflow aortic side to the inflow side of valve leaflets have identified side-specific differences in VEC expression for various calcification inhibitors, suggesting that there is heterogeneity in the VEC phenotype [Simmons et al., 2005]. Another type of phenotypical heterogeneity is addressed in this study. Despite the observed phenotypic differences between VECs and vascular ECs, little work has been done to characterize where VECs fit in the spectrum of known EC behavioral subpopulations.
Comparing VECs to known EC phenotypes serves to provide a frame of reference for potential differentiation studies needed in the future to provide an EC population similar to VECs for tissue engineering applications.

Vascular ECs can be generally categorized into three phenotypes: tip, stalk and phalanx. Here, these populations will be referred to as angiogenic ECs and nonangiogenic ECs. Angiogenic ECs (tip/stalk) are stimulated by a chemical signal, usually vascular endothelial growth factor (VEGF) [Gerhardt et al., 2003], to begin migration, proliferation and sprout formation towards the source of the signal. However, the more recently identified nonangiogenic ECs (phalanx) do not respond in the same manner, preferring to remain in the lumen of the parent vessel [Mazzone et al., 2009; Blancas et al., 2012]. Since the VEC layer in normal valves also appears to be generally nonmigratory [Chalajour et al., 2004], the potential for similarity between VECs and nonangiogenic ECs merits investigation.

Previous studies have shown that the ECs located in the interior of normal heart valves (within the leaflet as opposed to the surface) contain a population of lymphatic ECs (LECs) [Johnson and Blake, 1966; Kholová et al., 2013]. In pathological conditions, there is an increase of angiogenesis as well as lymphangiogenesis within the valves, possibly in response to a variety of inflammatory signals [Kholová et al., 2011, 2013]. However, it is unknown whether the LECs are restricted to the interior of the valve or if there is a population on the outside of the valve leaflets as well. Therefore, the purpose of this study was to characterize the population of VECs cultured from the surface of aortic valves with respect to a broad range of EC phenotypes, including angiogenic, nonangiogenic and lymphatic.

\section{Materials and Methods}

\section{Cell Isolation and Purification}

VECs were harvested from aortic valve leaflets and aortic ECs (AECs) from aortic root tissues dissected from fresh porcine adult hearts (6 months old). Tissues were purchased from the commercial abattoirs Fisher Ham and Meats (Spring, Tex., USA) and Animal Technologies (Tyler, Tex., USA). After dissection, tissues were digested in a collagenase II/dispase solution for $1 \mathrm{~h}$ at $37^{\circ} \mathrm{C}$ to loosen the ECs. Next, VECs and AECs were gently scraped from the surface of valve leaflets and the lumen of aortic tissues, respectively. The collected cells were then cultured on gelatin-coated flasks in EGM-2 basal growth media using bullet kit (Lonza, Walkersville, Md., USA) plus $1 \%$ penicillin/streptomycin (Lonza). At passage P1, isolated ECs were purified for CD31+ populations using CD31 antibody (Millipore, Temecula, Calif., USA) conjugated CELLection 
magnetic sorting beads (Invitrogen/Life Technologies, Carlsbad, Calif., USA). Cells were sorted once when freshly isolated, and once more if thawed from frozen stock. VECs were used between passages $\mathrm{P} 1$ and P5, and AECs were used between passages P1 and P6.

\section{Flow Cytometry Analysis}

VECs and AECs were analyzed via flow cytometry for various EC and LEC markers. Cells were harvested using Cell Dissociation Buffer (Life Technologies) and fixed in $4 \%$ formaldehyde for $1 \mathrm{~h}$ at $4^{\circ} \mathrm{C}$. For intracellular staining, cells were additionally treated with ice-cold methanol $\left(-20^{\circ} \mathrm{C}\right)$ for $1 \mathrm{~h}$ at $4^{\circ} \mathrm{C}$. Primary antibodies for EC markers CD31 (MCA1746 clone LCI-1, AbD Serotec, Raleigh, N.C., USA) and Flt-1 (sc-31173 clone N-16, Santa Cruz, Dallas, Tex., USA), tip-cell/angiogenesis-related markers CXCR4 (ab2074, Abcam, Cambridge, Mass., USA) and DLL4 (ab7280, Abcam), and lymphatic markers Prox1 (ab33219 clone 5G10, Abcam) and LYVE1 (ab33682, Abcam) were all used at a concentration of $1 \mu \mathrm{g}$ per million cells in a staining buffer containing $1 \%$ bovine serum albumin (BSA) in phosphate-buffered saline (PBS). Primary antibody incubation occurred at room temperature for $30 \mathrm{~min}$, with agitation using a vortex at $15 \mathrm{~min}$. Secondary antibodies conjugated with Alexa Fluor 488/647 (Invitrogen/Life Technologies and Abcam) were also used at $1 \mu \mathrm{g}$ per million cells in a staining buffer containing $1 \%$ BSA in PBS. Secondary antibody incubation occurred at room temperature for $30 \mathrm{~min}$, with agitation using a vortex at $15 \mathrm{~min}$. All samples $(\mathrm{n}=3-6$ biological replicates) were run on a BD FACSCanto II (BD Biosciences, Franklin Lakes, N.J., USA) and analyzed using FlowJo software (FlowJo, Ashland, Oreg., USA).

\section{Transwell Migration Assay}

Migration toward an angiogenic stimulus was evaluated by the use of a transwell migration assay. Fluoroblok 8- $\mu \mathrm{m}$ transwell inserts (Corning, Corning, N.Y., USA) were used in a 24-well plate. Inside, the transwells were seeded with $2 \times 10^{5}$ cells (either AECs or VECs) in $300 \mu \mathrm{l}$ of EGM-2 (Lonza). The well underneath the transwell insert contained $500 \mu \mathrm{l}$ of EGM-2 with an additional 50 $\mathrm{ng} / \mathrm{ml}$ of VEGF (R\&D Systems, Minneapolis, Minn., USA). After cells were incubated for $24 \mathrm{~h}$, the transwells were fixed in $4 \%$ formaldehyde and stained with DAPI. The undersides of the inserts were then imaged using a Zeiss LSM 510 LIVE5 confocal microscope (Zeiss, Oberkochen, Germany). The image field was $968 \times$ $968 \mu \mathrm{m}$. Intact nuclei were counted and presented as the mean \pm the standard error of the mean $(n=3-4$ biological replicates tested in triplicate). JMP statistical software (SAS, Cary, N.C., USA) was used to compare differences between cell counts using a two-tailed Student $\mathrm{t}$ test. $\mathrm{p}$ values $<0.05$ were considered statistically significant.

\section{Fluorescence Immunocytochemical Staining}

To assess the in situ expression of EC and LEC markers, VECs and AECs cultured on gelatin-coated chamber well slides $(n=3$ biological replicates) were fixed with $4 \%$ paraformaldehyde for 30 min at room temperature and blocked with $2 \%$ FBS in PBS overnight at $4^{\circ} \mathrm{C}$. Primary antibodies for CD31 (ab28364, Abcam), Flt1 (sc-31173, Santa Cruz), CXCR4 (ab2074, Abcam) and LYVE1 (ab33682, Abcam) were used at 1:100 dilutions in PBS and incubated onto samples overnight. Secondary antibodies conjugated with Alexa Fluor 488/555/633 (Invitrogen/Life Technologies) were used after primary antibody incubation. Fluorescent imaging was performed using a Zeiss LIVE5 confocal microscope.

\section{Tissue Immunohistochemical Staining}

Aortic valve leaflets were harvested from 10 porcine hearts purchased from the commercial abattoirs Fisher Ham and Meats, and Animal Technologies. The leaflets were fixed in 10\% neutral-buffered formalin for 3 days and subsequently dehydrated in an increasing series of alcohol/ $\mathrm{dH}_{2} \mathrm{O}$ solutions (Flex 100, Richard Allen Scientific/Thermo Fisher Scientific, Waltham, Mass., USA), a 50:50 Flex:xylene solution (histological grade xylene; Sigma Aldrich, St. Louis, Mo., USA), and finally $100 \%$ xylene. The leaflets were then soaked in paraffin for $3 \mathrm{~h}$ at $59^{\circ} \mathrm{C}$ and subsequently embedded in paraffin blocks. A Leica RM2135 microtome (Leica Biosystems, Nussloch, Germany) was used to section the leaflets into slices 5 - $\mu$ m-thick slices, which were mounted on glass slides. Primary antibodies for CD31 (ab119341, Abcam) and LYVE1 (ab33682, Abcam) were used at 1:100 dilutions in 0.5\% BSA in PBS and incubated at room temperature for $3 \mathrm{~h}$. Secondary antibodies conjugated with Alexa Fluor 488/568 (Invitrogen/Life Technologies) were incubated for $1 \mathrm{~h}$ at room temperature. The slides were sealed with Fluoromount-G (Southern Biotech, Birmingham, Ala., USA). Fluorescent imaging was performed using a Zeiss LIVE5 confocal microscope. Chromogenic immunohistochemical staining [Connell et al., 2015] was used to provide confirmation for the staining of LYVE1 as well as to demonstrate another LEC marker, Prox1, at a dilution of 1:100. To provide microstructural context for the immunostaining, additional tissue sections were stained with Movat's pentachrome as previously described [Connell et al., 2015]. Leaflets from 4 hearts were used for immunofluorescent staining, whereas leaflets from the other 6 hearts were used for immunohistochemical and Movat staining.

\section{Results}

\section{Demonstration of Angiogenic Phenotypic \\ Characteristics}

Flow cytometry analysis demonstrated that VECs expressed almost double the amount of Flt-1 compared to AECs, measured as 74 versus $38 \%$, respectively (fig. 1a). The angiogenic EC markers CXCR4 and DLL4 both showed over $90 \%$ expression on AECs, whereas VECs showed much lower levels, with only $35 \%$ CXCR4+ and $47 \%$ DLL4+, indicating a less angiogenic phenotype. Im-

Fig. 1. VECs display fewer proangiogenic markers than AECs. a Flow cytometry analysis of both cell populations demonstrates that AECs express higher levels of the proangiogenic/tip EC markers CXCR4 and DLL4 than VECs. Representative results from 3-6 samples. Additionally, VECs express higher levels of Flt-1, which is common in nonangiogenic/phalanx ECs as it dampens the migratory signal of VEGF. CD31 is used as a pan-endothelial check to ensure both populations consist of only ECs. b Immunofluorescence comparison of VEC and AEC expression of CD31, Flt-1 and CXCR4 (representative results from 3 samples); the results confirmed the flow cytometry results. Scale bars $=50 \mu \mathrm{m}$.

(For figure see next page.) 


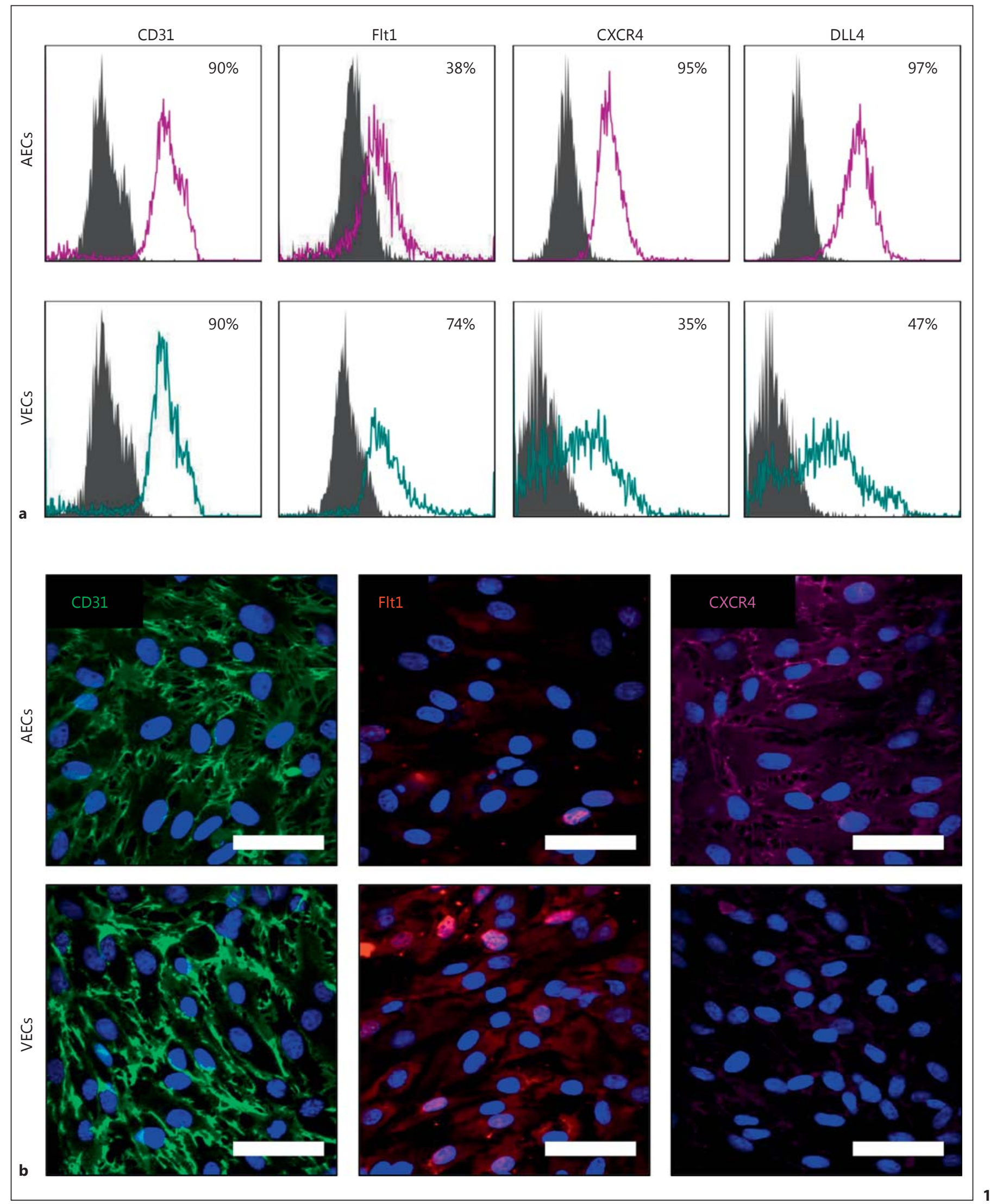




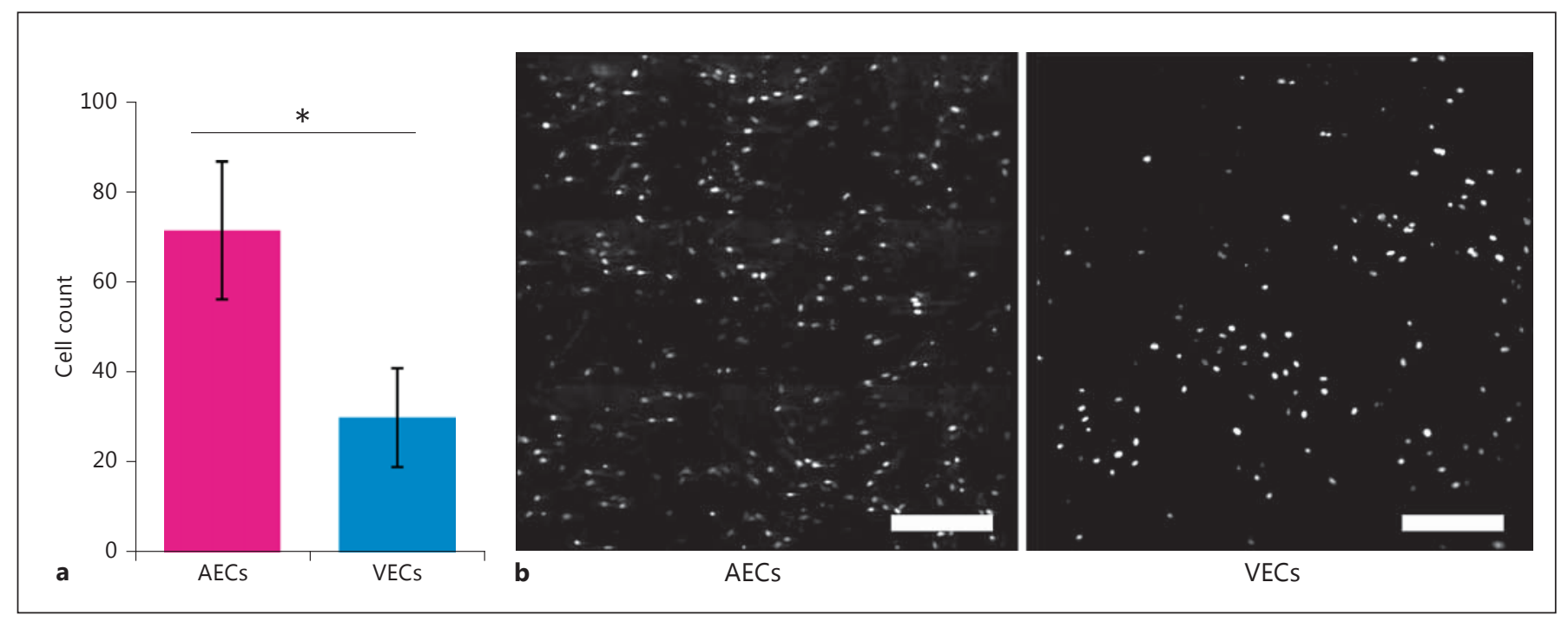

Fig. 2. VECs display largely nonmigratory behavior. a AECs demonstrated a significantly higher migration towards an angiogenic stimulus (EGM-2 plus an additional $50 \mathrm{ng} / \mathrm{ml}$ of VEGF). ${ }^{*} \mathrm{p}<0.05$, $n=12-16$. Error bars represent the standard error of the mean. b Representative images of AECs and VECs that have migrated through the transwell membrane. Scale bars $=200 \mu \mathrm{m}$.
Fig. 3. A population of LECs are present in VECs. a Flow cytometry analysis of lymphatic markers LYVE1 and Prox1 in AECs and VECs $(n=3)$. There is a clear population of LYVE1+/Prox 1+ cells in the VECs that is absent in the AECs. b Immunofluorescent stain of AECs and VECs on 1\% gelatin. Cells stained with LYVE1 are green and DAPI-stained nuclei are blue $(\mathrm{n}=3)$. Scale bars $=50 \mu \mathrm{m}$.

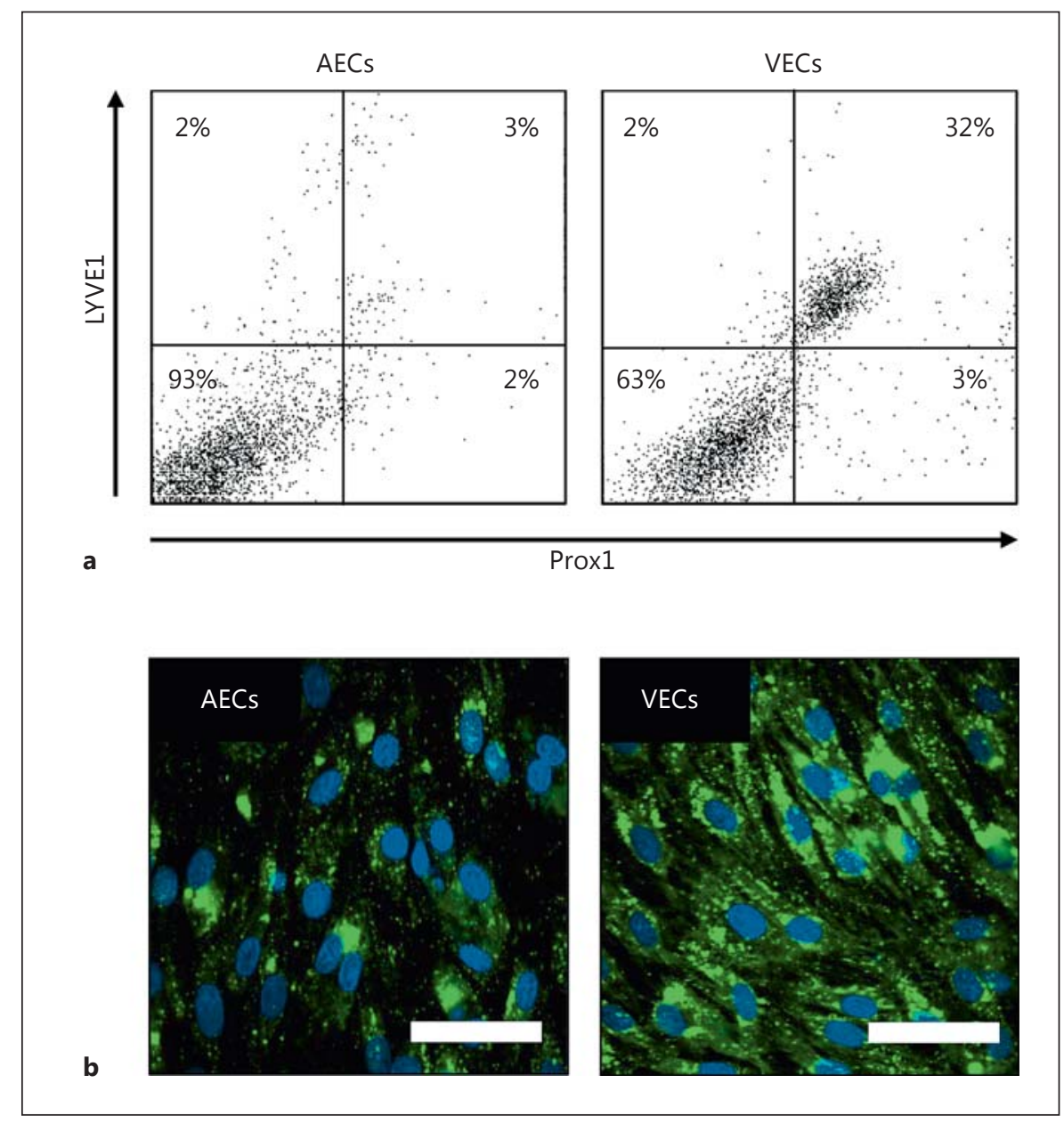


Fig. 4. LEC markers have different expression patterns on the valve leaflets. a Movat's pentachrome staining depicting the layered structure of the valve leaflet. $\mathrm{V}=$ Ventricularis; $\mathrm{S}=$ spongiosa; $\mathrm{F}=$ fibrosa. As seen with immunofluorescent staining, LYVE1 expression (red arrowheads) is localized to the endothelial layer; secondary control (b); LYVE1 stain (c). Prox1 is expressed throughout all layers of the leaflet; secondary control (d); Proxl stain (e). Representative samples from 6 replicates. f Immunofluorescent staining indicated that the LEC marker LYVE1 (green) was colocalized with CD31 (red) on the surface of the valves. The staining was particularly strong on the ventricular-facing surface (inset). Cell nuclei counterstained with DAPI are blue. Representative samples from 4 replicates. Scale bars $=100 \mu \mathrm{m}$.

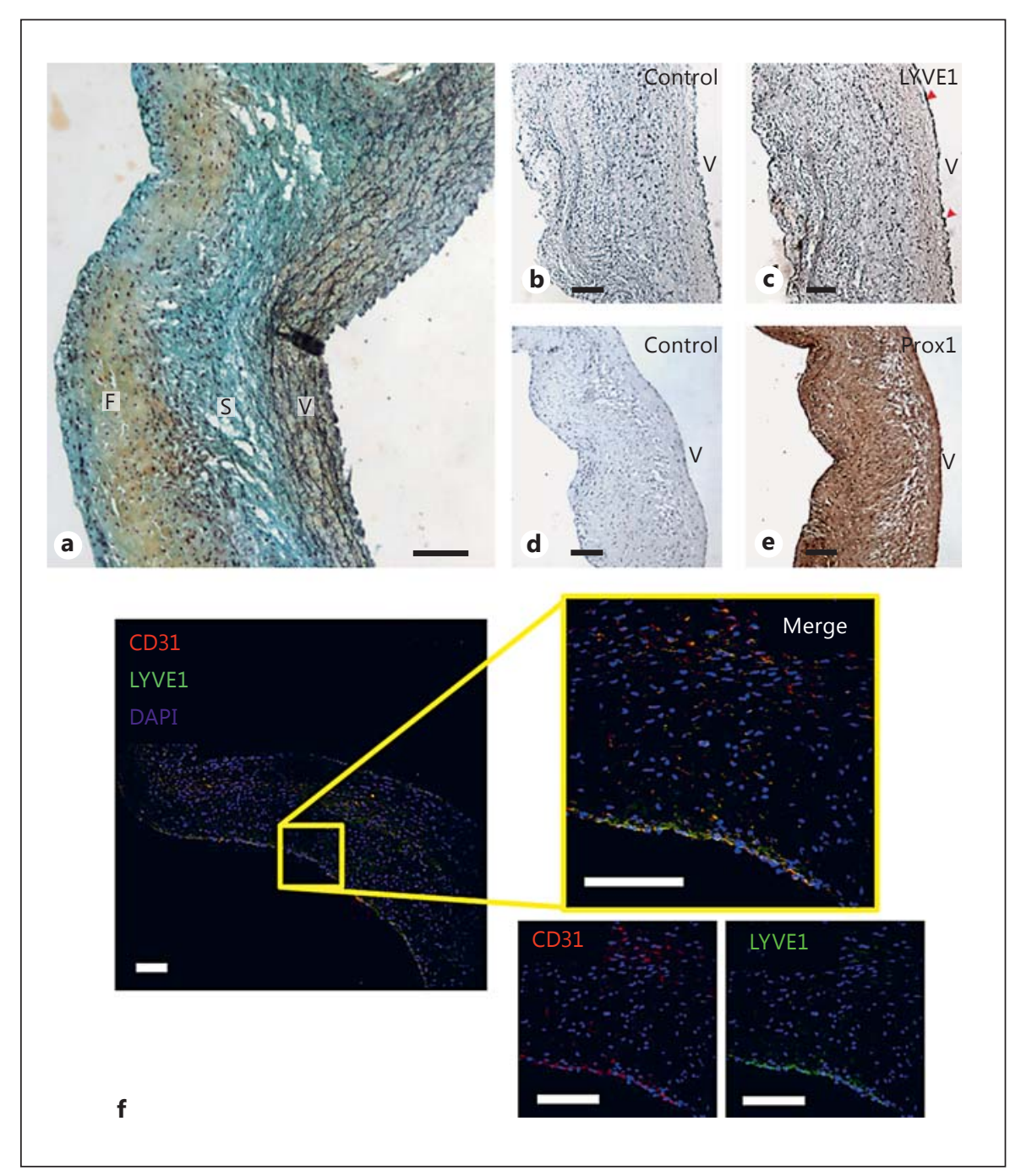

munofluorescent staining also verified the high expression of CXCR4 and low expression of Flt-1 in AECs and the opposite result in VECs (fig. 1b). Pan-endothelial marker CD31 expression was consistently high $(>90 \%)$ in both cell populations, indicating the high level of EC purity after isolation and sorting.

\section{Migration toward Angiogenic Stimulus}

To compare the migratory potential of the AECs and VECs, a transwell assay was performed utilizing VEGF as the migratory signal (fig. 2). After $24 \mathrm{~h}$, the AECs demonstrated significantly greater migration with a mean of 71.5 \pm 11.0 cells per image field, while the mean number of VECs per image field was $30.0 \pm 15.3(\mathrm{p}=0.032)$. The behavioral difference between the two populations corresponded to the high expression levels of angiogenic/tip EC markers

Phenotypes and Heterogeneity in Heart Valve Surface Endothelium found on the AECs and the lower levels of the same markers, as well as the high expression of Flt-1, on the VECs.

\section{Demonstration of Lymphatic Endothelial Phenotypic \\ Characteristics}

For further characterization of the molecular and behavioral phenotype of VECs, the possibility of EC heterogeneity was considered. Both cell types were examined for expression of the LEC markers LYVE1 and Prox1 (fig. 3a). An appreciable population of VECs, approximately $30 \%$, was LYVE1+/Prox1+, whereas these markers were not present in AECs. Immunofluorescent staining verified the difference in LYVE1 expression between VECs and AECs (fig. 3b). Another well-established lymphatic marker, Flt-4/VEGFR3, was not expressed on either the VECs or AECs (data not shown), but it is likely that the lack of 
expression in this study was due to culture conditions not conducive to its expression. Flt- 4 is a receptor for VEGF isoforms C and D [Mäkinen et al., 2001], and EGM-2 medium only contains VEGF isoform A.

\section{Localization of Lymphatic Characteristics within Valvular Tissue}

The distribution of LECs on the surface of valves appears to correspond to the location of VECs (fig. 4). Chromogenic staining of LYVE1 (fig. 4b, c), as well as fluorescent staining of CD31 and LYVE1 (fig. 4f) showed that they co-localize together on the valve surface with both markers showing the strongest signal along the ventricularis. Chromogenic staining for Prox1 showed expression throughout the entire leaflet (fig. $4 \mathrm{~d}, \mathrm{e}$ ).

\section{Discussion}

Although VECs demonstrate the capacity to transdifferentiate into a mesenchymal phenotype as well as differences in osteogenic markers under stress in comparison to vascular ECs [Butcher et al., 2006], these valvular cells are often treated as a uniform population with general pan-endothelial traits, such as the expression of CD31 and von Willebrand factor and the production of basement membrane extracellular matrix [Balaoing et al., 2014]. Although that generalization is not without merit, this study evaluated the complexities and heterogeneity unique to this cell population and showed the existence of two distinct populations on the valve surface endothelium: nonangiogenic VECs and LEC-like cells. The understanding of such nuances will be fundamental to future study of normal and pathological heart valve function. In addition, as progress is made toward generating engineered tissues that are suitable for valve replacements, it becomes necessary to ensure that the population of cells seeded within these engineered tissues represents a phenotype that resembles the in vivo characteristics as closely as possible. Valve cells are particularly challenging in this regard, since harvesting cells from a patient is considered to be extremely invasive if not impossible. Therefore, a suitable VEC facsimile would need to be derived from a different source, such as bone marrow stem cells [Rath et al., 2015], peripheral blood endothelial progenitor cells (EPCs), umbilical cord blood EPCs, or possibly induced pluripotent stem cells.

A pronounced expression of Flt-1, as observed here in VECs, has become a hallmark trait of nonangiogenic/phalanx ECs, and may be a marker for the stability of the en- dothelial layer atop valves. Flt-1 appears to mitigate angiogenic signaling by serving as a sort of 'trough' for excess VEGF [Gille et al., 2001]. VECs expressed not only high levels of Flt-1, but also showed reduced migration towards angiogenic stimulus, both of which are traits observed in nonangiogenic ECs [Mazzone et al., 2009; Blancas et al., 2012]. These aspects of nonangiogenic behavior by VECs was further supported by their low-to-moderate expression of angiogenic EC markers CXCR4 and DLL4, which are known to play an important role in angiogenesis and are found on tip ECs [Hellström et al., 2007; Strasser et al., 2010]. As expected, AECs expressed low levels of Flt-1 [Krueger et al., 2011] and high expression of DLL4 and CXCR4. With respect to recapitulating these VEC characteristics in a tissue-engineered valve, future investigators could perform directed differentiation towards a nonangiogenic EC phenotype; this approach has already been developed using embryonic stem cells as a source population [Blancas et al., 2012] and the scheme could be modified to accommodate other cell populations. Having a seeded EC population with the ability to remain stationary after implantation would be critical for the success of a tissue-engineered or bioprosthetic valve. Not only would this EC population be subject to dynamic shear stresses from the unique blood flow environment surrounding heart valves, but the cells would also need the capacity to withstand potential migratory signals that could result from the implantation procedure itself. If the VEC standin population could be derived from autologous cells such as EPCs, the transplanted valve would have a barrier that prevents or minimizes the immune response.

The question of whether or not LECs exist on the outside of the leaflets in the VEC layer was motivated by the finding of lymphatic vessels inside valve leaflets [Johnson and Blake, 1966; Kholová et al., 2013], although the function of lymphatics within heart valves has not been fully elucidated. As the lymphatic system transports immune system-related cells, it is possible that lymphatic vessels in the interior of the valves serve as a prophylactic measure to prevent, or subsequently combat, valve disease. Valve thickening has been attributed in part to changes in lymph flow [Miller, 2011]. Calcified areas within aortic valves, especially in cases of stenosis, show an increase in the number of lymphatic vessels and blood vessels, likely in response to inflammation [Kholová et al., 2011; Syväranta et al., 2012]. A common way to distinguish LECs from vascular ECs is by examining the expression of two or more known lymphatic markers coexpressed in a single population [Oliver, 2004]. Markers include LYVE1, a hyaluronan receptor [Banerji et al., 1999], and Prox1, a tran- 
scription factor that is considered to be the best functional marker for LECs [Hong et al., 2002]. An LYVE1+/ Prox 1+ population was found within the VECs, indicating the presence of lymphatic-like cells on the outside of the valves in the VEC layer, although further studies are necessary to determine what role they play in normal and abnormal valves. The strong expression of LYVE1 on the surface, colocalized with CD31, merits further investigation. It is possible that the expression of LEC markers on the leaflets may decrease once the cells are harvested and cultured, indicating that there is an element of phenotype plasticity that could shed light on VEC and LEC function. A possible purpose for the valve LECs is that they serve as a 'reserve' population of LECs that can migrate into the valve when needed, thereby giving a boost to an immune response to valve disease.

Another interesting finding was the expression of Prox1 throughout the entire leaflet. This result is consistent with the in vivo lymphatic vasculature, in which Prox1 is found largely in the valves, while LYVE1 remains in the lumen [Sabine et al., 2012]. Indeed, LYVE1 is downregulated in fluid shear stress, which explains its expression on the lumen only [Sabine et al., 2012], which makes its expression on the leaflet surface, an area known to undergo fluid stress, unexpected. Future studies into the expression of LYVE1 and Prox1 under shear stress conditions, and potentially other endothelial lineage-related markers, in VECs and valve interstitial cells in normal and pathological states could provide more insight into the inflammatory response at various stages of disease progression.

In summary, the ECs comprising the outer layer of the aortic valve leaflets are a heterogeneous population that includes LEC-like cells and minimally or nonangiogenic/ phalanx phenotypes. In the future, it will be important to assess how these cells promote overall valve health or are affected by valve disease. Furthermore, this VEC heterogeneity has implications for heart valve tissue engineering. Although this study is preliminary in nature, it highlights how the in vivo valve cell populations should be examined just as carefully as the mechanical properties of the valve leaflets in which they reside. The consideration of both factors is necessary for maximizing the success of bioengineered constructs and therapies. In heart valve therapy specifically, understanding the changes in composition of the valve cell populations and leaflet matrix that occur as a patient ages or as a result of pathological causes can shed light on how the constituents work together to form a functional tissue. This information would not only aid in the development of suitable engineered replacements, but also in how to prevent or minimize complications postimplantation.

\section{Acknowledgements}

The authors express special thanks to Patrick Connell, who provided additional porcine aortic valve leaflets for histological analysis/staining. This work was supported by the National Institutes of Health (R01 HL107765), the March of Dimes (6-FY13-86), and an American Heart Association predoctoral fellowship (for L.R.B.).

\section{Disclosure Statement}

The authors have no conflicts of interest to declare.

\section{References}

Balaoing, L.R., A.D. Post, H. Liu, K.T. Minn, K.J. Grande-Allen (2014) Age-related changes in aortic valve hemostatic protein regulation. Arterioscler Thromb Vasc Biol 34: 72-80.

Banerji, S., J. Ni, S.-X. Wang, S. Clasper, J. Su, R. Tammi, M. Jones, D.G. Jackson (1999) LYVE1 , a new homologue of the CD44 glycoprotein, is a lymph-specific receptor for hyaluronan. J Cell Biol 144: 789-801.

Blancas, A.A., L.E. Wong, D.E. Glaser, K.E. McCloskey (2012) Specialized tip/stalk-like and phalanx-like endothelial cells from embryonic stem cells. Stem Cell Dev 22: 1398-1407.

Butcher, J.T., A.M. Penrod, A.J. García, R.M. Nerem (2004) Unique morphology and focal adhesion development of valvular endothelial cells in static and fluid flow environments.
Arterioscler Thromb Vasc Biol 24: 14291434.

Butcher, J.T., S. Tressel, T. Johnson, D. Turner, G. Sorescu, H. Jo, R.M. Nerem (2006) Transcriptional profiles of valvular and vascular endothelial cells reveal phenotypic differences: influence of shear stress. Arterioscler Thromb Vasc Biol 26: 69-77.

Chalajour, F., H. Treede, A. Ebrahimnejad, H. Lauke, H. Reichenspurner, S. Ergun (2004) Angiogenic activation of valvular endothelial cells in aortic valve stenosis. Exp Cell Res 298: 455-464.

Connell, P.S., A.F. Azimuddin, S.E. Kim, F. Ramirez, M.S. Jackson, S.H. Little, K.J. Grande-Allen (2015) Regurgitation hemodynamics alone cause mitral valve remodeling characteristic of clinical disease states in vitro. Ann Biomed Eng, Epub ahead of print.

Deck, J.D. (1986) Endothelial cell orientation on aortic valve leaflets. Cardiovasc Res 20: 760 767.

El-Hamamsy, I., A.H. Chester, M.H. Yacoub (2010) Cellular regulation of the structure and function of aortic valves. J Adv Res 1: 5-12.

Farrar, E.J., J.T. Butcher (2014) Heterogeneous susceptibility of valve endothelial cells to mesenchymal transformation in response to TNFa. Ann Biomed Eng 42: 149-161.

-Gerhardt, H., M. Golding, M. Fruttiger, C. Ruhrberg, A. Lundkvist, A. Abramsson, M. Jeltsch, C. Mitchell, K. Alitalo, D. Shima (2003) VEGF guides angiogenic sprouting utilizing endothelial tip cell filopodia. J Cell Biol 161: 1163-1177.
Phenotypes and Heterogeneity in Heart Valve Surface Endothelium
Cells Tissues Organs 2015-16;201:268-276 DOI: $10.1159 / 000444446$ 
Gille, H., J. Kowalski, B. Li, J. LeCouter, B. Moffat, T.F. Zioncheck, N. Pelletier, N. Ferrara (2001) Analysis of biological effects and signaling properties of Flt-1 (VEGFR-1) and KDR (VEGFR-2): a reassessment using novel receptor-specific vascular endothelial growth factor mutants. J Biol Chem 276: 3222-3230.

Hellström, M., L.-K. Phng, J.J. Hofmann, E. Wallgard, L. Coultas, P. Lindblom, J. Alva, A.-K. Nilsson, L. Karlsson, N. Gaiano (2007) Dll4 signalling through Notch1 regulates formation of tip cells during angiogenesis. Nature 445: 776-780.

Hong, Y.K., N. Harvey, Y.H. Noh, V. Schacht, S. Hirakawa, M. Detmar, G. Oliver (2002) Prox1 is a master control gene in the program specifying lymphatic endothelial cell fate. Dev Dyn 225: 351-357.

-Johnson, R.A., T.M. Blake (1966) Lymphatics of the heart. Circulation 33: 137-142.

Kholová, I., G. Dragneva, P. Čermáková, S. Laidinen, N. Kaskenpää, T. Hazes, E. Čermáková, I. Šteiner, S. Ylä-Herttuala (2011) Lymphatic vasculature is increased in heart valves, ischaemic and inflamed hearts and in cholesterolrich and calcified atherosclerotic lesions. Eur J Clin Invest 41: 487-497.

Kholová, I., G. Dragneva, S. Ylä-Herttuala (2013) The lymphatics in normal and pathological heart valves; in Karunamuni G (ed): The Cardiac Lymphatic System. New York, Springer, pp 63-71.

Krueger, J., D. Liu, K. Scholz, A. Zimmer, Y. Shi, C. Klein, A. Siekmann, S. Schulte-Merker, M. Cudmore, A. Ahmed (2011) Flt1 acts as a negative regulator of tip cell formation and branching morphogenesis in the zebrafish embryo. Development 138: 2111-2120.
Mahler, G.J., E.J. Farrar, J.T. Butcher (2013) Inflammatory cytokines promote mesenchymal transformation in embryonic and adult valve endothelial cells. Arterioscler Thromb Vasc Biol 33: 121-130.

Mäkinen, T., T. Veikkola, S. Mustjoki, T. Karpanen, B. Catimel, E.C. Nice, L. Wise, A. Mercer, H. Kowalski, D. Kerjaschki (2001) Isolated lymphatic endothelial cells transduce growth, survival and migratory signals via the VEGF-C/D receptor VEGFR-3. EMBO J 20: 4762-4773.

Mazzone, M., D. Dettori, R. Leite de Oliveira, S. Loges, T. Schmidt, B. Jonckx, Y.-M.Tian, A.A. Lanahan, P. Pollard, C. Ruiz de Almodovar (2009) Heterozygous deficiency of PHD2 restores tumor oxygenation and inhibits metastasis via endothelial normalization. Cell 136 839-851.

Miller, A. (2011) The grossly invisible and generally ignored lymphatics of the mammalian heart. Med Hypotheses 76: 604-606.

Oliver, G. (2004) Lymphatic vasculature development. Nat Rev Immunol 4: 35-45.

- Paranya, G., S. Vineberg, E. Dvorin, S. Kaushal, S.J. Roth, E. Rabkin, F.J. Schoen, J. Bischoff (2001) Aortic valve endothelial cells undergo transforming growth factor- $\beta$-mediated and non-transforming growth factor- $\beta$-mediated transdifferentiation in vitro. Am J Pathol 159: 1335-1343.

Paruchuri, S., J.-H. Yang, E. Aikawa, J.M. MeleroMartin, Z.A. Khan, S. Loukogeorgakis, F.J. Schoen, J. Bischoff (2006) Human pulmonary valve progenitor cells exhibit endothelial/ mesenchymal plasticity in response to vascular endothelial growth factor-A and transforming growth factor- $\beta 2$. Circ Res 99: 861869.
Puperi, D.S., L.R. Balaoing, R.W. O’Connell, J.L. West, K.J. Grande-Allen (2015) 3-dimensional spatially organized PEG-based hydrogels for an aortic valve co-culture model. Biomaterials 67: 354-364.

Rath, S., M. Salinas, A.G. Villegas, S. Ramaswamy (2015) Differentiation and distribution of marrow stem cells in flex-flow environments demonstrate support of the valvular phenotype. PloS One 10: e0141802.

Sabine, A., Y. Agalarov, H. Maby-El Hajjami, M. Jaquet, R. Hägerling, C. Pollmann, D. Bebber, A. Pfenniger, N. Miura, O. Dormond (2012) Mechanotransduction, PROX1, and FOXC2 cooperate to control connexin 37 and calcineurin during lymphatic-valve formation. Dev Cell 22: 430-445.

Simmons, C.A., G.R. Grant, E. Manduchi, P.F. Davies (2005) Spatial heterogeneity of endothelial phenotypes correlates with side-specific vulnerability to calcification in normal porcine aortic valves. Circ Res 96: 792-799.

-Strasser, G.A., J.S. Kaminker, M. Tessier-Lavigne (2010) Microarray analysis of retinal endothelial tip cells identifies CXCR4 as a mediator of tip cell morphology and branching. Blood 115: 5102-5110.

Syväranta, S., S. Helske, J. Lappalainen, M. Kupari, P.T. Kovanen (2012) Lymphangiogenesis in aortic valve stenosis - novel regulatory roles for valvular myofibroblasts and mast cells. Atherosclerosis 221: 366-374.

Wiltz, D., C.A. Arevalos, L.R. Balaoing, A.A. Blancas, M.C. Sapp, X. Zhang, K.J. GrandeAllen (2013) Extracellular matrix organization, structure, and function; in Aikawa $\mathrm{E}$ (ed): Calcific Aortic Valve Disease. Rijeka, InTech. http://www.intechopen.com/books/ calcific-aortic-valve-disease/extracellularmatrix-organization-structure-and-function. 